\title{
Parameters Effecting on Head Brown Rice Recovery and Energy Consumption of Rubber Roll and Stone Disk Dehusking
}

\author{
Nguyen Duc Canh ${ }^{1}$, Nguyen Thanh Nghi $^{1}$ and Nguyen Van Hung ${ }^{2}$ \\ 1. Center for Agricultural Energy and Machinery, Nong Lam University, Ho Chi Minh City 700000, Vietnam \\ 2. Crop and Environmental Science Division, International Rice Research Institute, Metro Manila 1301, Philippines
}

\begin{abstract}
Application of improper methods on rice processing affects rice quality and head rice recovery. In Vietnam, paddy with different moisture contents (from 13\% to 17\%) is dehusked by both rubber roll and stone disk. Thus, objective of this research was to evaluate the technical and economic aspects of the two methods. Optimization was conducted with 20 experiments for input factors (moisture content) and output factors (head brown rice recovery, specific energy consumption). Besides, other factors were also monitored, such as the gap between the two disks, speed of disk and roll, and pressure of rubber roll on paddy. Test results showed that the maximum value of head brown rice $(77.4 \%)$ and the minimum value of specific energy consumption $(0.66 \mathrm{kWh} / \mathrm{ton})$ corresponding to moisture content of paddy of $13.7 \%$ for stone disk dehusker. At similar moisture content (13.7\%), head brown rice recovery and specific energy consumption were $77.2 \%$ and $1.04 \mathrm{kWh} /$ ton for rubber roll dehusker, respectively. As the result, specific energy consumption of rubber roll dehusker was higher than that of stone disk dehusker, corresponding to the higher dehusking efficiency.
\end{abstract}

Key words: Paddy moisture content, rubber roll dehusker, stone disk dehusker, head mill rice recovery, energy consumption.

\section{Introduction}

Vietnam is one of the major rice producers with around 44.1 million tons of paddy harvested annually [1]. In the Mekong River Delta of Vietnam, paddy is usually dehusked at two different levels of moisture content and based on the two processes-wet husking of paddy at $17 \%$ wet basic moisture content (MCwb) and dry husking of paddy at $14 \%$ MCwb [2]. Husking at moisture content of $16 \%-17 \%$ (wet paddy) is mainly for export purpose. Wet paddy purchased from traders is dehusked and then brown rice after milling is stored. When there is an order for exporting, brown rice with high moisture content will be dried, whitened, polished and packed. On the other hand, husking at moisture content of $13 \%-14 \%$ (dried paddy) is mainly for local use purpose [2]. This method has been applied in countries with a developed rice processing industry, such as America and Thailand [3]. Fresh paddy is dried (mechanical drying or sun drying) to $13 \%-14 \%$, then dehusked, whitened, polished and distributed.

In each kind of process, paddy is dehusked using a stone disc, a rubber roller or a combined disc and roller type. Each process and equipment has its own advantages and disadvantages. For example, rubber roller has more dehusking efficiency, but has more power consumption compared to the stone disc. [3].

However, there is limited research on comparison of these dehusking technologies, especially for the current applications in Mekong Delta. This study focused on the effect of the two levels of paddy moisture content on brown head rice recovery and specific power consumption.

\footnotetext{
Corresponding author: Nguyen Thanh Nghi, Ph.D.,
} research field: agricultural engineering. 


\section{Materials and Methods}

Experimental method was applied to evaluate the effects of factors on milling quality. Each experiment was conducted independently to evaluate on level of effect.

The experiment was designed with two input factors: paddy moisture $(\omega)$, and distance between discs $(\delta)$ for disc dehusker or pressing force of two rollers for roller dehusker $(f)$. Output factors were head rice recovery $(E)$ and specific energy consumption (Ar) (Fig. 1). Numbers of experiments were 10 for each treatment based on the experiment design method of orthogonal order 2 [4, 5]. Experiment was randomly designed using Statgraphics software program.

According to Rulten [6], head rice recovery and dehusking efficiency were calculated by the Eq. (1) and Eq. (2):

$$
\begin{aligned}
& \text { Head rice recovery }=\frac{\text { weight of head rice }}{\text { weight of clean paddy }} \\
& \text { Dehusking efficiency }=\frac{\text { weight of dehusked paddy }}{\text { weight of clean paddy }}
\end{aligned}
$$

Jasmine 85 variety with long and aromatic grain was selected, because it was popularly planted in Mekong Delta.

After harvesting, paddy was mechanically dried using reversible air flatbed dryer SRA-500 (Fig. 2) with capacity of $500 \mathrm{~kg} / \mathrm{batch}$ designed and fabricated by Center for Agricultural Energy and Machinery (drying at low temperature) [7]. Initial MCwb of paddy was $22.8 \%$. The paddy MCwb was determined using an oven method by Hien et al. [7].

According to Afzalinia et al. [8], head rice recovery is unaffected when MCwb of paddy is lower than $13 \%$. Moreover, rice will be reduced inquality or damaged when it is stored with MCwb over 17\% [2]. Thus, this study only researched paddy with MCwb from $13 \%-17 \%$. After drying, the MCwb of paddy was shown in Table 1.
After drying, paddy was cleaned using a cleaner with the cleanness of about 96\%-98\%.

In this study, a CL 1000 dehusker with rubber roller (Fig. 3) made by Vinappro company and a disc dehusker made by Phuoc Thanh Company were used. According to Satake [9], diameter, length and speed of roller were usually $150-250 \mathrm{~mm}, 60-250 \mathrm{~mm}$ and 14 $\mathrm{m} / \mathrm{s}$, respectively. Unlike the original CL 1000, it is additionally installed with a programmable logic controller (PLC) equipment for measuring and controlling press forces between the two husking rollers.

In addition, other equipment and instruments, such as stop watch, scales and power meter, were used to determine experimental parameters.

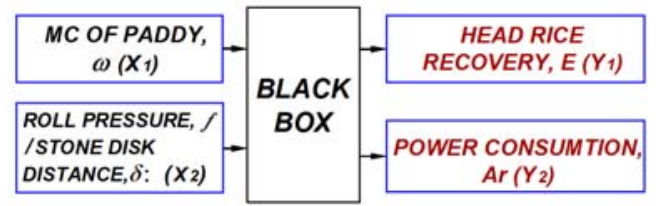

Fig. 1 Schematic problem of optimization.

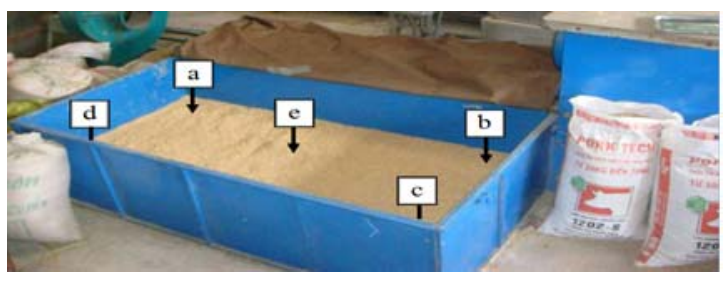

Fig. 2 SRA-500 dryer and sampling position.

Table 1 MCwb test results of paddy samples.

\begin{tabular}{lllllll}
\hline MCwb & Position & a & c & d & e & Average \\
\hline $13 \%-14 \%$ & 13.8 & 13.8 & 13.7 & 13.5 & 13.7 & 13.7 \\
$\approx 15 \%$ & 15.2 & 15.3 & 15.0 & 14.9 & 15.0 & 15.1 \\
$16 \%-17 \%$ & 16.5 & 16.6 & 16.5 & 16.3 & 16.3 & 16.4 \\
\hline
\end{tabular}

a, b, c, d and e presented for positions in the bin of dryer (Fig. 2 ). As the result, paddy was tested at three levels of moisture content $(\omega)$ : $13.7 \%, 15.1 \%$ and $16.4 \%$.
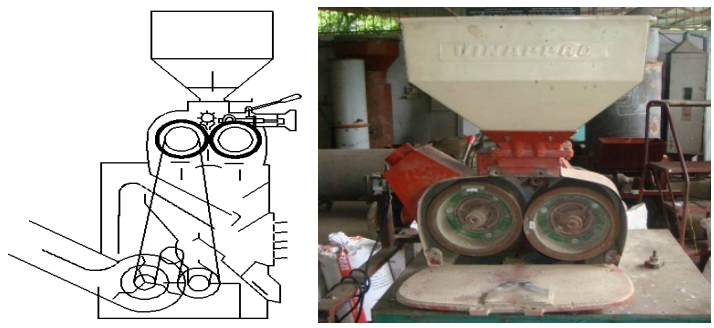

Fig. 3 Dehusker CL 1000 . 


\section{Results and Discussion}

Test result of single factor experiment showed that paddy moisture $(\omega)$, distance between discs $(\delta)$ for disc dehusker and pressing force of two rollers $(f)$ have the highest level of effect on head rice recovery $(E)$ and specific power consumption $\left(A_{r}\right)$. Test result of single factor experiments also showed the limitation and variation of input factors (Table 2).

\subsection{Experiment with Disc Dehusker}

Experimental matrix of 10 experiments and test results were shown in Table 3.

Based on the results, the highest of brown head rice recovery and the lowest of specific power consumption corresponded to low level of paddy moisture content $\left(X_{1}=-1\right)$ and high level of distance between two disc $\left(X_{2}=1\right)$. It is explained that when the distance between discs is big, paddy is not rubbed by the surface of two discs, paddy is mainly rubbed by paddy and disc, and paddy and paddy. On the other hand, with low moisture content, the husk is crispy and the rice kernel is harder. Thus, the husk is dehusked easier and less broken brown rice.

Regression function of brown head rice recovery was analyzed with a significance level of $5 \%$ as Eq. (3):

$$
\begin{aligned}
E= & -131.831+17.2592 \times \omega+33.1059 \times \delta- \\
& 0.5428 \times \omega^{2}-0.5278 \times \omega \delta-2.2393 \times \delta^{2}
\end{aligned}
$$

As shown in Fig. 4, shape of function $(E)$ is a part of a convex parabola. The maximum value of this function corresponds with the edge area of $\omega$ and $\delta$. It is proportional to $\delta$, inverse proportional to $\omega$ and has the maximum at two diagonal corners. The maximun value is at the intersection between two edge area of $\omega$ $=13.7 \%$ and $\delta=6 \mathrm{~mm}$ with a respond $E$ surface. The maximum value of $E$ is $E_{\max }=77.4 \%$.

Similarly, the result of regression analysis for specific energy consumption function was shown as Eq. (4):

$$
\begin{aligned}
A r= & 14.0816-1.0581 \times \omega-2.1593 \times \delta+0.0321 \times \\
& \omega^{2}+0.0389 \times \omega \delta+0.1336 \times \delta^{2}
\end{aligned}
$$

Based on this regression equation, the factor $\delta$ mostly affects on $A_{r}$. Function $A_{r}$ is inverse proportional to $\delta$ and proportional to $\omega$.

Table 2. Variation of input factors.

\begin{tabular}{lll}
\hline Parameters & Low level & High level \\
\hline$\omega(\%)$ & $13 \%-14 \%$ & $16 \%-17 \%$ \\
$\delta(\mathrm{mm})$ & 4 & 6 \\
$f(\mathrm{~N} / \mathrm{mm})$ & 6.5 & 9.0 \\
\hline
\end{tabular}

Table 3 Test result for disc dehusker (encoded).

\begin{tabular}{lllll}
\hline No. & $X_{1}$ & $X_{2}$ & $Y_{1}(\%)$ & $Y_{2}(\mathrm{kWh} /$ ton $)$ \\
\hline 1 & 1 & -1 & 76.4 & 1.4 \\
2 & -1 & 0 & 76.1 & 0.9 \\
3 & 1 & 1 & 71.4 & 1.0 \\
4 & -1 & -1 & 70.5 & 1.3 \\
5 & 0 & 0 & 74.9 & 0.9 \\
6 & 1 & 0 & 71.2 & 1.1 \\
7 & -1 & 1 & 75.3 & 0.8 \\
8 & 0 & 1 & 75.3 & 0.8 \\
9 & 0 & 0 & 75.1 & 0.9 \\
10 & 0 & -1 & 69.5 & 1.3 \\
\hline
\end{tabular}

$X_{1}=$ moisture content of paddy (\%); $X_{2}=$ stone disk distance $(\mathrm{mm}) ; Y_{1}=$ head rice recovery (\%); $Y_{2}=$ energy consumption $(\mathrm{kWh} / \mathrm{ton})$

\section{Estimated Response Surface}

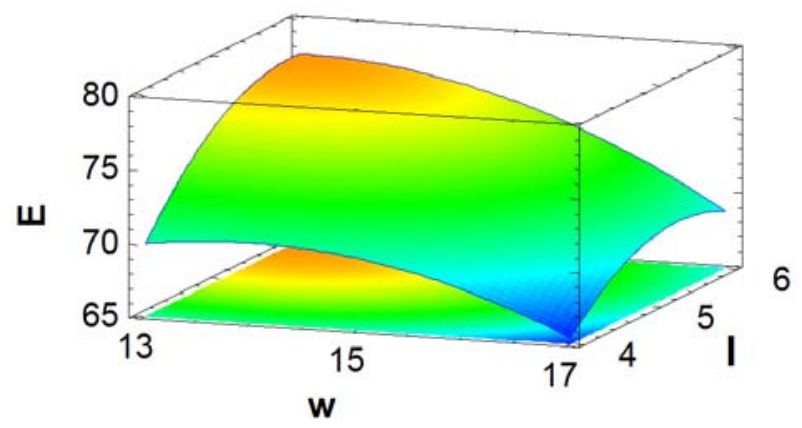

Fig. 4 Graph of $E$ function of disc dehusker.

\section{Estimated Response Surface}

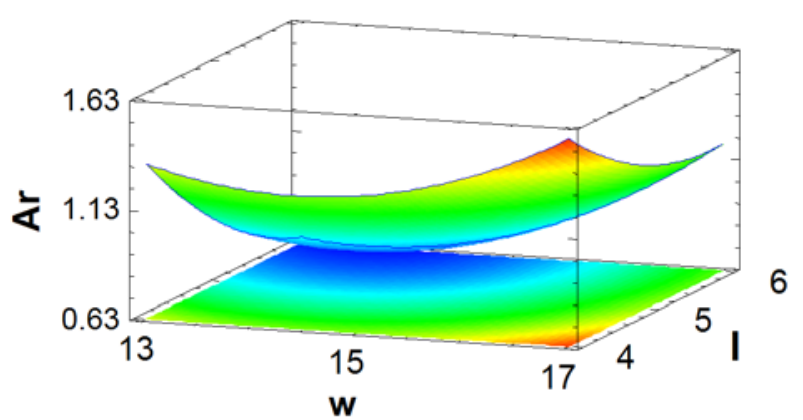

Fig. 5 Graph of $A_{r}$ function of disc dehusker. 
Shape of $A_{r}$ function is a part of a concave parabolic (Fig. 5). The maximum value is at edge areas of $\omega$ and $\delta$. The function is proportional to $\delta$, inverse proportional to $\omega$ and has the maximum at two diagonal corners of edge area. The minimum value at lowest area of $\omega$ and $\delta$. The minimum value is $A_{\text {rmin }}=0.6 \mathrm{kWh} /$ ton, corresponding to $\omega=13.7 \%$ and $\delta=6 \mathrm{~mm}$.

\subsection{Test Result for Rubber Roller Dehusker}

Test results of press force of rollers were shown in Fig. 6 and the test result for rubber roller dehusker was in Table 4.

When paddy is low moisture content $\left(X_{1}=-1\right)$ and the press force between rollers is light $\left(X_{2}=-1\right)$, the brown head rice recovery is the maximum and specific power consumption is the lowest. It is explained that the low press force between rollers causes low press force on paddy grain.

On the other hand, when low moisture content of paddy, the husk is crispy and rice kernel is harder. Thus, the husk is easier to be detached and broken brown rice is less.

As shown in regression analysis (Fig. 7), the shape of $E$ function is a part of a convex paraboloid, expressed by Eq. (5):

$$
\begin{aligned}
E= & -166.812+23.1957 \times \omega+23.6801 \times f- \\
& 0.6847 \times \omega^{2}-0.5630 \times \omega f-1.1602 \times f^{2}
\end{aligned}
$$

The maximum value is at the edge area of $\omega$ and $f$. While because it is inverse proportional to both $\omega$ and $f$, the maximum value is at two diagonal corners of $\omega$ and $f$. The maximum valu is $E_{\max }=77.3 \%$, corresponding to $\omega=13.7 \%$ and $f=6.5 \mathrm{~N} / \mathrm{mm}$.

In the contract, shape of $A r$ function is a part of a concave paraboloid (Fig. 8), expressed as Eq. (6):

$$
\begin{aligned}
A r= & -0.2252-0.3624 \times \omega+0.5503 \times f+ \\
& 0.0667 \times \omega f-0.0779 \times f^{2}
\end{aligned}
$$

The maximum value at the edge positions of edge area of $\omega$ and $f$. The minimum value is at low edge area of $\omega$ and $f$. Thus, the minimum value is $A_{\text {rmin }}=1.0 \mathrm{kWh} /$ ton, corresponding to $\omega=13.7 \%$ and $f=6.5 \mathrm{~N} / \mathrm{mm}$.

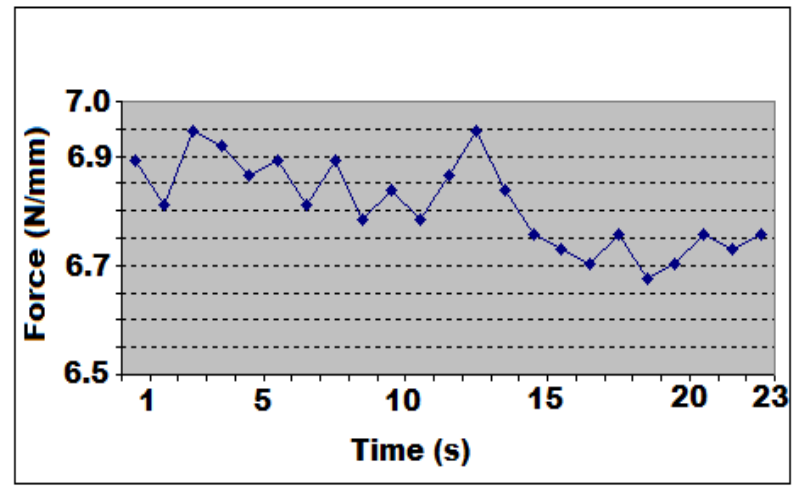

Fig. 6 Graph of press force of rollers.

Table 4 Experiment results for rubber roller dehusker.

\begin{tabular}{lllll}
\hline No. & $X_{1}$ & $X_{2}$ & $Y_{1}(\%)$ & $Y_{2}(\mathrm{kWh} /$ ton $)$ \\
\hline 1 & 0 & -1 & 77.2 & 1.1 \\
2 & -1 & 0 & 76.3 & 1.5 \\
3 & 1 & 1 & 65.4 & 2.3 \\
4 & 1 & 0 & 72.1 & 1.9 \\
5 & 0 & 0 & 75.4 & 1.7 \\
6 & -1 & -1 & 77.3 & 1.0 \\
7 & -1 & 1 & 72.4 & 1.7 \\
8 & 0 & 1 & 70.1 & 2.0 \\
9 & 0 & 0 & 75.2 & 1.7 \\
10 & 1 & -1 & 74.1 & 1.3 \\
\hline
\end{tabular}

$X_{1}=$ moisture content of paddy (\%); $X_{2}=$ roll pressure $(\mathrm{N} / \mathrm{mm}) ;$ $Y_{1}=$ head rice recovery (\%); $Y_{2}=$ energy consumption (kWh/ton).

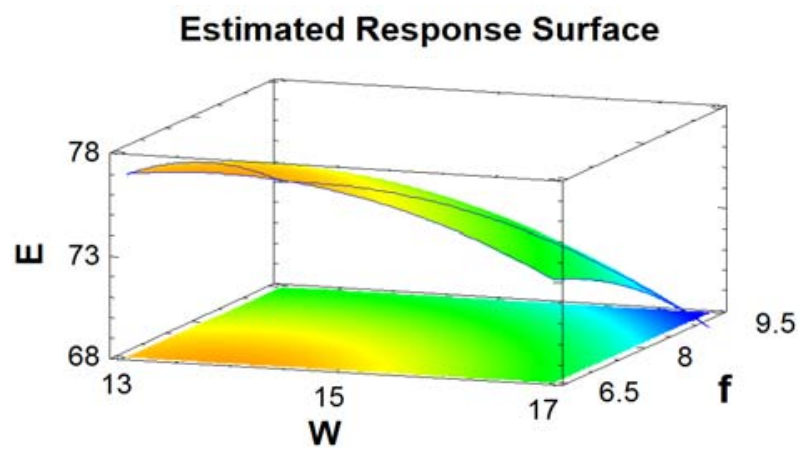

Fig. 7 Graph of $E$ function of rubber roller dehusker.

Estimated Response Surface

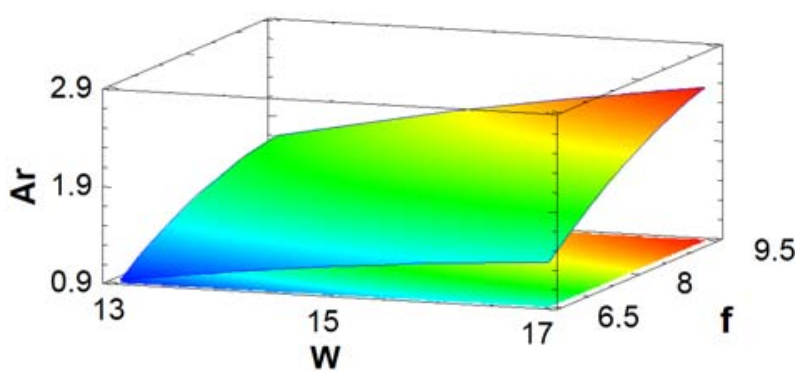

Fig. 8 Graph of $A r$ function of rubber roller dehusker. 
The difference of brown head rice recovery between the two methods is insignificant with high value of $E_{\max }^{M B V L}=76.3 \%$ and $E_{\max }^{M B V D}=77.4 \%$, corresponding to paddy with moisture content of $13.7 \%$. It is confirmed that paddy is dehusked at moisture content of $13 \%-14 \%$ resulting a high brown head rice recovery, corresponding to high white head rice recovery.

\subsection{Optimum Analysis}

In rice processing, the optimum value corresponds to the lowest specific power consumption and the highest brown head rice recovery. It is difficult to achieve both these objectives. Depending on each specific condition, the priority is for brown head rice recovery $(E)$ or specific power consumption (Ar). Thus, the optimum analysis was solved using Matlab program.

3.3.1 Optimum Function of $E=E_{\max }$

In case that the objective of head rice recovery is prioritized and specific power consumption is insignificant, the objective function is as Eq. (7):

$$
\begin{aligned}
E= & -131.831+17.2592 \times \omega+33.1059 \times \delta-0.5428 \\
& \times \omega^{2}-0.5278 \times \omega \delta-2.2393 \times \delta^{2} \Rightarrow \max (7)
\end{aligned}
$$

with boundary condition (limitation of factors):

$$
13.7<\omega<16.4 ; 4<\delta<6
$$

and solved result:

$$
\omega=13.7000, \delta=5.7775 ; \quad \text { fval }=77.4882
$$

Based on the result, with moisture content of paddy at $\omega=13.7 \%$ and the distance between discs at $\delta=5.8$ $\mathrm{mm}$, the $E$ function is the maximum with $E_{\max }=$ $77.5 \%$ (Fig. 9) and head rice recovery $(H)=52.8 \%$. Respond surface of $E$ function in experimental area is a part of convex paraboloid, the maximum area is in the intersection of two edge surfaces $\omega=13.7 \%$ and $\delta$ $=6.0 \mathrm{~mm}$ and $A_{r}=0.68 \mathrm{kWh} / \mathrm{ton}$.

\subsubsection{Optimization of the Function $A_{r}=A_{r m i n}$}

In case that specific energy consumption factor is prioritized, the objective function is as Eq. (8):

$$
\begin{aligned}
A_{r}= & 14.0816-1.0581 \times \omega-2.1593 \times \delta+0.0321 \times \\
& \omega^{2}+0.0389 \times \omega \delta+0.1336 \times \delta^{2} \Rightarrow \min \quad(8)
\end{aligned}
$$

with boundary condition:

$$
13.7<\omega<16.4 ; 4<\delta<6
$$

and sovled result:

$$
\omega=13.7000, \delta=6.0000 ; \text { fval }=0.6619
$$

thus, $A_{r}=A_{r \min }=0.66 \mathrm{kWh} / \mathrm{ton}$ at $\omega=13.7 \%$ and $\delta=$ $6.0 \mathrm{~mm}$, then $E=77.4 \% ; H=50.0 \%$ (Fig. 10).

\subsubsection{Multi-objective Optimization}

If the objective function is as Eq. (9):

$$
\begin{aligned}
A_{r}= & 14.0816-1.0581 \times \omega-2.1593 \times \delta+0.0321 \times \\
& \omega^{2}+0.0389 \times \omega \delta+0.1336 \times \delta^{2} \Rightarrow \min \text { (9) }
\end{aligned}
$$

with boundary condition:

$13.7<\omega<16.4 ; \quad 4.0<\delta<6.0,75 \%<E<78 \%$ and solved result:

$$
\omega=13.7000, \delta=6.000 ; \text { fval }=0.6619
$$

This result is similar to the optimum $A_{r}=A_{r m i n}$.

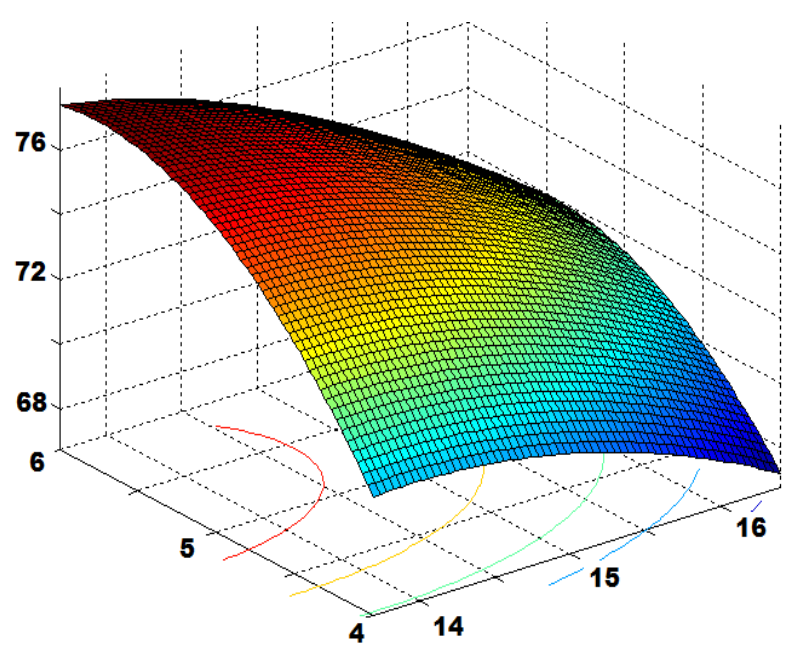

Fig. 9 Graph of optimum function $E$.

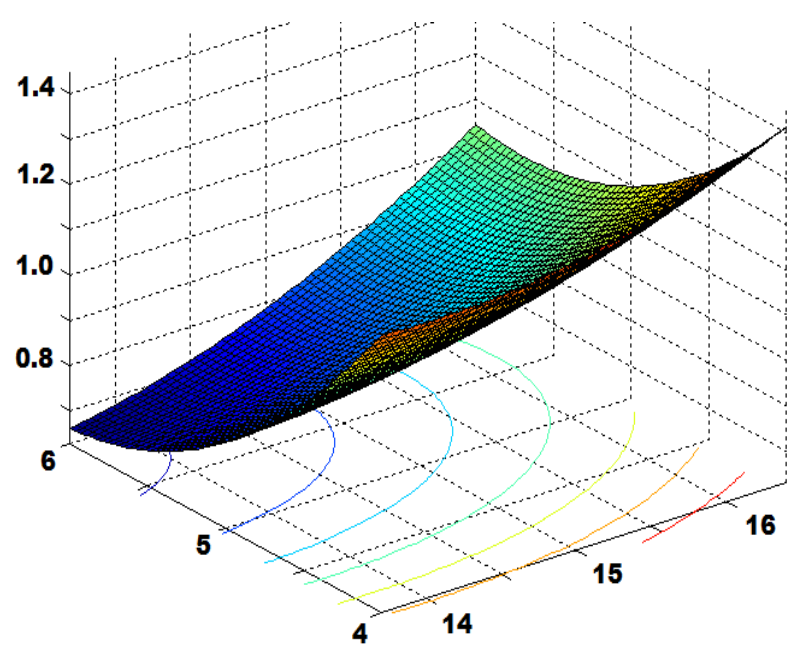

Fig. 10 Graph of optimum function $\mathrm{Ar}$. 


\section{Conclusions}

The optimization research illustrated that paddy is dehusked at MCwb of $13 \%-14 \%$ with a high brown head rice recovery, corresponding to high white head rice recovery.

The rollers press the grain and create frictions in the opposite direction on each grain, specific energy consumption of roll dehusker with $1.02 \mathrm{kWh} / \mathrm{ton}$ of paddy is nearly double than of disc dehusker. On the other hand, dehusking efficiency and capacity of the rubber roller dehusker are much higher than that of disc dehusker with same dimension. Dehusking efficiency of rubber roller dehusker (80\%) is higher than that of the disc dehusker (55\%).

\section{References}

[1] General Statistical Office (GSO). 2013. Statistical Data 2013. Hanoi, Vietnam: GSO.

[2] Xuan, N. V., Khanh, T. V., Canh, N. D., and Tuan, T. V.
2011. "Research on the Effect of Paddy Moisture Content on Milling Quality (Head Rice Recovery) in Rice Processing.” In Proceedings of 2011 Scientific Conference on Postharvest Loss Reduction, 636-44.

[3] Khanh, T. V. 2010. Rice Processing Technology on Rice Processing of Vietnam. Vietnam: Agricultural Publisher, 96-126.

[4] Tri, B. M. 2006. Statistical Probability and Experimental Design. Ho Chi Minh City, Vietnam: Scientific and Technical Publisher.

[5] Canh, N. 2011. Experimental Design. Ho Chi Minh City: Vietnam National University.

[6] Rulten, H. V. 1990. Technology on Rice Processing, translated by Nguyen, H. H. Hanoi, Vietnam: Institute of Tools and Mechanization, 15-25.

[7] Hien, P. H., Xuan, N. V., Tam, N. H., Ban, L. V., and Vinh, T. 2000. Grain Dryers in Vietnam. Ho Chi Minh City, Vietnam: Vietnam Agricultural Publisher.

[8] Afzalinia, S., Shaker, M., and Zare, E. 2004. "Comparison of Different Rice Milling Methods." Canadian Biosystems Engineering 46 (3): 63-6.

[9] Satake, T. 1990. Modern Rice-Milling Technology. Tokyo: University of Tokyo Press. 